Bull. Soc. math. France

130 (1), 2002, p. 35-48

\title{
AN APPROXIMATION PROPERTY OF QUADRATIC IRRATIONALS
}

\author{
By TAKaO Komatsu
}

Dedicated to Professor Iekata Shiokawa on the occasion of his 60th birthday

\begin{abstract}
Let $\alpha>1$ be irrational. Several authors studied the numbers
\end{abstract}

$$
\ell^{m}(\alpha)=\inf \left\{|y|: y \in \Lambda_{m}, y \neq 0\right\},
$$

where $m$ is a positive integer and $\Lambda_{m}$ denotes the set of all real numbers of the form $y=\epsilon_{0} \alpha^{n}+\epsilon_{1} \alpha^{n-1}+\cdots+\epsilon_{n-1} \alpha+\epsilon_{n}$ with restricted integer coefficients $\left|\epsilon_{i}\right| \leq m$. The value of $\ell^{1}(\alpha)$ was determined for many particular Pisot numbers and $\ell^{m}(\alpha)$ for the golden number. In this paper the value of $\ell^{m}(\alpha)$ is determined for irrational numbers $\alpha$, satisfying $\alpha^{2}=a \alpha \pm 1$ with a positive integer $a$.

RÉSUMÉ (Une approximation des irrationnels quadratiques). - Soit $\alpha>1$ un irrationnel. Plusieurs auteurs ont étudié les nombres

$$
\ell^{m}(\alpha)=\inf \left\{|y|: y \in \Lambda_{m}, y \neq 0\right\},
$$

où $m$ est un entier positif et $\Lambda_{m}$ est l'ensemble de tous les réels de la forme $y=$ $\epsilon_{0} \alpha^{n}+\epsilon_{1} \alpha^{n-1}+\cdots+\epsilon_{n-1} \alpha+\epsilon_{n}$ avec des $\left|\epsilon_{i}\right| \leq m$ entiers. La valeur de $\ell^{1}(\alpha)$ a été précisée pour beaucoup de nombres de Pisot et $\ell^{m}(\alpha)$ pour le nombre d'or. Dans cet article, on détermine $\ell^{m}(\alpha)$ lorsque $\alpha$ est un irrationnel qui satisfait $\alpha^{2}=a \alpha \pm 1$ avec $a$ entier positif.

Texte reçu le 16 juin 2000, révisé le 18 décembre 2000, accepté le 11 avril 2001 Takao Komatsu, Faculty of Education, Mie University, Mie 514-8507 (Japan) E-mail : komatsu@edu.mie-u.ac.jp 2000 Mathematics Subject Classification. - 11A63, 11J04, 11J70.

Key words and phrases. - Approximation property, quadratic irrationals, continued fractions. 


\section{Introduction}

Let $\alpha$ be a positive real number and an integer $m \geq 1$. Denote by $\Lambda=\Lambda_{m}$ the set of all real numbers $y$ having at least one representation of the form

$$
y=\epsilon_{0} \alpha^{n}+\epsilon_{1} \alpha^{n-1}+\cdots+\epsilon_{n-1} \alpha+\epsilon_{n}
$$

with some positive integer $n$ and $\left|\epsilon_{i}\right| \leq m, \epsilon_{i} \in \mathbb{Z}(i=0,1, \ldots, n)$. Set

$$
\ell^{m}(\alpha)=\inf \left\{|y|: y \in \Lambda_{m}, y \neq 0\right\} .
$$

Several authors studied the numbers $\ell^{m}(\alpha)$. The value of $\ell^{1}(\alpha)$ was determined for many particular Pisot numbers (see [1], [3], [4], [5], [6], [7], [8]) and $\ell^{m}(\alpha)$ for the golden number (see [8]). In this paper the value of $\ell^{m}(\alpha)$ is determined for two kinds of irrational numbers:

$$
\begin{aligned}
& \alpha=\frac{1}{2}\left(a+\sqrt{a^{2}+4}\right)=[a ; a, a, \ldots] \quad(a \geq 1) \quad \text { and } \\
& \alpha=\frac{1}{2}\left(a+\sqrt{a^{2}-4}\right)=[a-1 ; 1, a-2,1, a-2, \ldots] \quad(a \geq 3) .
\end{aligned}
$$

We shall prove the following two theorems.

TheOREM 1.1. - Let $\alpha=\frac{1}{2}\left(a+\sqrt{a^{2}+4}\right)(a \geq 1)$. If

$$
\alpha^{k}(\alpha-1)<m \leq \alpha^{k+1}(\alpha-1)
$$

for some integer $k \geq-1$, then

$$
\ell^{m}(\alpha)=\left|q_{k} \alpha-p_{k}\right|
$$

TheOREM 1.2. - Let $\alpha=\frac{1}{2}\left(a+\sqrt{a^{2}-4}\right) \quad(a \geq 3)$.

- If for some non-negative integer $i$,

$$
\alpha^{i}(\alpha-a+1)<m \leq \alpha^{i}(a-2)
$$

then

- If for some non-negative integer $i$,

$$
\ell^{m}(\alpha)=\left|q_{2 i-1} \alpha-p_{2 i-1}\right| .
$$

$$
\alpha^{i}(a-2)<m<\alpha^{i+1}(\alpha-a+1)
$$

then

$$
\ell^{m}(\alpha)=\left|q_{2 i} \alpha-p_{2 i}\right|
$$

In addition to prove these two theorems, we shall show how to find a representation form $y=\epsilon_{0} \alpha^{n}+\epsilon_{1} \alpha^{n-1}+\cdots+\epsilon_{n-1} \alpha+\epsilon_{n}$ which gives $\ell^{m}(\alpha)=$ $\left|q_{k} \alpha-p_{k}\right|$.

TOME $130-2002-\mathrm{N}^{\mathrm{O}} 1$ 


\section{General sketch}

If $\alpha$ is a root of the quadratic equation, then any of the form

$$
\epsilon_{0} \alpha^{n}+\epsilon_{1} \alpha^{n-1}+\cdots+\epsilon_{n-1} \alpha+\epsilon_{n} \quad(n \geq 2)
$$

can be reduced to the form $q \alpha-p$ for some integers $q$ and $p$. We can set $\alpha>1$. For, $\ell^{m}(\alpha)=0$ if $0<\alpha<1 ; \ell^{m}(\alpha)=1$ if $\alpha=1$. Concerning the linear form $q \alpha-p$, the following approximation theorem is well-known (see Thm. 5E (ii) in [10], e.g.).

Theorem A. - If $k \geq 1,0<q \leq q_{k}$ and $p / q \neq p_{k} / q_{k}, p / q \neq p_{k-1} / q_{k-1}$, then

$$
\left|q_{k-1} \alpha-p_{k-1}\right|<|q \alpha-p| \text {. }
$$

Here, $p_{k} / q_{k}=\left[a_{0} ; a_{1}, \ldots, a_{k}\right]$ denotes the $k$-th convergent of the continued fraction expansion of $\alpha, \alpha=\left[a_{0} ; a_{1}, a_{2}, \ldots\right]$. Namely,

$$
\begin{array}{rlrl}
\alpha & =a_{0}+\frac{1}{\alpha_{1}}, & a_{0}=\lfloor\alpha\rfloor, & \\
\alpha_{n}=a_{n}+\frac{1}{\alpha_{n+1}}, & a_{n}=\left\lfloor\alpha_{n}\right\rfloor \quad(n \geq 1)
\end{array}
$$

and

$$
\begin{array}{llll}
p_{k}=a_{k} p_{k-1}+p_{k-2} & (k \geq 0), & p_{-1}=1, & p_{-2}=0, \\
q_{k}=a_{k} q_{k-1}+q_{k-2} & (k \geq 0), & q_{-1}=0, & q_{-2}=1 .
\end{array}
$$

Therefore, this kind of problems is equivalent to how to find the least $m$ with $\left|\epsilon_{i}\right| \leq m, \epsilon_{i} \in \mathbb{Z}(0 \leq i \leq n)$, satisfying

$$
\epsilon_{0} \alpha^{n}+\epsilon_{1} \alpha^{n-1}+\cdots+\epsilon_{n-1} \alpha+\epsilon_{n}=q \alpha-p= \pm\left(q_{k} \alpha-p_{k}\right)
$$

for a fixed integer $k$. In other words, $\ell^{m}(\alpha)$ is equal a priori to some $\left|q_{k} \alpha-p_{k}\right|$ for every $m$. Namely, for any positive integer $m$ there exists an integer $k$ such that for some $y=y_{-1}, y_{0}, \ldots, y_{k}$ we have

$$
y_{-1}=\left|q_{-1} \alpha-p_{-1}\right|, \quad y_{0}=\left|q_{0} \alpha-p_{0}\right|, \ldots, \quad y_{k}=\left|q_{k} \alpha-p_{k}\right|,
$$

but $y \neq\left|q_{k+1} \alpha-p_{k+1}\right|$ for any $y$. From the result of van Ravenstein [9], the integer $q$ satisfying $\ell^{m}(\alpha)=q \alpha-p$ should be one of the first values

$$
\left\{(-1)^{n} j q_{n} / q_{n+1}\right\} q_{n+1} \quad(j=1,2, \ldots),
$$

and the integer $p$ be its counterpart. Notice that larger $m$ becomes, more choices each $\epsilon_{i}$ can have. So, always $\ell^{m}(\alpha) \leq \ell^{m+1}(\alpha)$ for every $m$. If $q>q_{k+1}$ then from our decision of $m$ (see (7) below) we could choose some $y$ so that $y=\left|q_{k+1} \alpha-p_{k+1}\right|$ because

$$
|q| s_{n-1}+|p| s_{n}>q_{k+1} s_{n-1}+p_{k+1} s_{n} .
$$

BULletin DE LA SOCIÉtÉ MATHÉMATIQUE DE FRANCE 
Hence, it is sufficient to consider the integers $q$ with $q_{k}<|q|<q_{k+1}$. But, by Theorem A always $|q \alpha-p|>\left|q_{k} \alpha-p_{k}\right|$ holds for such $q$ 's.

Suppose that $\alpha$ is the larger root of the quadratic equation $x^{2}=a x+b$. Here, $a, b \in \mathbb{Z}$ because both $q$ and $p$ are integers. Notice that $x^{2}-a x-b=$ $(x-\alpha)(x-\beta)$, where

$$
\alpha=\frac{a+\sqrt{a^{2}+4 b}}{2} \text { and } \beta=\frac{a-\sqrt{a^{2}+4 b}}{2},
$$

satisfying $\alpha+\beta=a, \alpha \beta=-b$. By $\alpha>1$, we have $a+b>1$, satisfying $a^{2}+4 b>0$. Put

$$
\alpha^{n}=s_{n} \alpha+t_{n} \quad \text { for } \quad n \geq 0 .
$$

LEMma 2.1. - One has

$$
\begin{gathered}
s_{n}=\frac{\alpha^{n}-\beta^{n}}{\alpha-\beta}, \quad t_{n}=b \frac{\alpha^{n-1}-\beta^{n-1}}{\alpha-\beta}=b s_{n-1} . \\
s_{n-1} s_{i}-s_{n} s_{i-1}=(-b)^{i-1} s_{n-i} \quad(i=1,2, \ldots, n) .
\end{gathered}
$$

Proof. - (1) Since the recurrence relation $r_{n}=a r_{n-1}+b r_{n-2}$ has the general solution

$$
r_{n}=\frac{\left(\alpha^{n-1}-\beta^{n-1}\right) r_{2}-\alpha \beta\left(\alpha^{n-2}-\beta^{n-2}\right) r_{1}}{\alpha-\beta},
$$

by using $s_{2}=a, s_{1}=1, t_{2}=b$ and $t_{1}=0$ we have

$$
s_{n}=\frac{\alpha^{n}-\beta^{n}}{\alpha-\beta} \quad \text { and } \quad t_{n}=b \frac{\alpha^{n-1}-\beta^{n-1}}{\alpha-\beta}=b s_{n-1} .
$$

(2) For $i=1,2, \ldots, n$

$$
\begin{aligned}
s_{n-1} s_{i}-s_{n} s_{i-1} & =s_{n-1}\left(a s_{i-1}+b s_{i-2}\right)-\left(a s_{n-1}+b s_{n-2}\right) s_{i-1} \\
& =-b\left(s_{n-2} s_{i-1}-s_{n-1} s_{i-2}\right) \\
& =(-b)^{2}\left(s_{n-3} s_{i-2}-s_{n-2} s_{i-3}\right)=\cdots \\
& =(-b)^{i-1}\left(s_{n-i} s_{1}-s_{n-i+1} s_{0}\right) \\
& =(-b)^{i-1} s_{n-i} .
\end{aligned}
$$

By using $s_{n}, y$ can be written as a linear form:

$$
\begin{aligned}
y= & \epsilon_{0} \alpha^{n}+\epsilon_{1} \alpha^{n-1}+\cdots+\epsilon_{n-1} \alpha+\epsilon_{n} \\
= & \left(\epsilon_{0} s_{n}+\epsilon_{1} s_{n-1}+\cdots+\epsilon_{n-2} s_{2}+\epsilon_{n-1} s_{1}\right) \alpha \\
& \quad+b\left(\epsilon_{0} s_{n-1}+\epsilon_{1} s_{n-2}+\cdots+\epsilon_{n-2} s_{1}\right)+\epsilon_{n} .
\end{aligned}
$$

TOME $130-2002-\mathrm{N}^{\mathrm{O}} 1$ 
Suppose that

$$
\begin{gathered}
\epsilon_{0} s_{n}+\epsilon_{1} s_{n-1}+\cdots+\epsilon_{n-2} s_{2}+\epsilon_{n-1} s_{1}=q_{k}, \\
b\left(\epsilon_{0} s_{n-1}+\epsilon_{1} s_{n-2}+\cdots+\epsilon_{n-2} s_{1}\right)+\epsilon_{n}=-p_{k}
\end{gathered}
$$

for some integer $k$. Otherwise, we interchange each sign of $\epsilon_{i}(i=0,1, \ldots, n)$. We shall find the least integer $m$ (say, $m^{\prime}$ ) which satisfies $\left|\epsilon_{i}\right| \leq m$ for all $i=0,1, \ldots, n$. By eliminating $\epsilon_{0}$ we have

$$
\begin{aligned}
& b\left(s_{n-1}^{2}-s_{n} s_{n-2}\right) \epsilon_{1}+b\left(s_{n-1} s_{n-2}-s_{n} s_{n-3}\right) \epsilon_{2} \\
& \quad+\cdots+b\left(s_{n-1} s_{2}-s_{n} s_{1}\right) \epsilon_{n-2}+b s_{n-1} \epsilon_{n-1}-s_{n} \epsilon_{n}=q_{k} b s_{n-1}+p_{k} s_{n} .
\end{aligned}
$$

By Lemma 2.1(2), we obtain

$$
\begin{aligned}
-(-b)^{n-1} s_{1} \epsilon_{1}-(-b)^{n-2} s_{2} \epsilon_{2} & -\cdots-(-b)^{2} s_{n-2} \epsilon_{n-2} \\
& +b s_{n-1} \epsilon_{n-1}-s_{n} \epsilon_{n}=q_{k} b s_{n-1}+p_{k} s_{n} .
\end{aligned}
$$

If $\operatorname{gcd}(a, b)=1$, then we have $\operatorname{gcd}\left(s_{i+1}, b s_{i}\right)=1(i \geq 1)$, yielding

$$
\operatorname{gcd}\left(b^{n-1} s_{1}, b^{n-2} s_{2}, \ldots, b s_{n-1}, s_{n}\right)=1 .
$$

In fact, $\operatorname{gcd}\left(s_{2}, b s_{1}\right)=\operatorname{gcd}(a, b)=1$. Assume that $\operatorname{gcd}\left(s_{n}, b s_{n-1}\right)=1$ for some $n$. Suppose that, however,

$$
\operatorname{gcd}\left(s_{n+1}, b s_{n}\right)=\operatorname{gcd}\left(a s_{n}+b s_{n-1}, b s_{n}\right)=c
$$

with $c \geq 2$. Since $c \mid b s_{n}$, we have for some divisor of $c$, say $c_{1}>1, c_{1} \mid b$ or $c_{1} \mid s_{n}$. If $c_{1} \mid b$ then by $c_{1} \mid s_{n+1}$ and $c_{1} \nmid a$ we have $c_{1} \mid s_{n}$, yielding $\operatorname{gcd}\left(s_{n}, b s_{n-1}\right)=c_{1}$. If $c_{1} \mid s_{n}$ then by $c_{1} \mid s_{n+1}$ we have $c_{1} \mid b s_{n-1}$, yielding $\operatorname{gcd}\left(s_{n}, b s_{n-1}\right)=c_{1}$, which is the contradiction again.

Therefore, the linear equation (5) is solvable in integers. We shall show the concrete step to obtain one of solutions in (5) in the following sections. $\left|\epsilon_{n}\right|$, $\left|\epsilon_{n-1}\right|, \ldots,\left|\epsilon_{1}\right|$ can be chosen as lexicographically minimal among those giving $\ell^{m}(\alpha)=\left|q_{k} \alpha-p_{k}\right|$.

After choosing the integers from $\epsilon_{n}$ to $\epsilon_{1}, \epsilon_{0}$ can be naturally determined as an integer if $\operatorname{gcd}(a, b)=1$. For, by $(3)$ and $(4)$

$$
\epsilon_{0}=\frac{q_{k}-\left(\epsilon_{1} s_{n-1}+\cdots+\epsilon_{n-1} s_{1}\right)}{s_{n}}=\frac{-p_{k}-b\left(\epsilon_{1} s_{n-2}+\cdots+\epsilon_{n-2} s_{1}\right)-\epsilon_{n}}{b s_{n-1}} .
$$

Since both of two fractions are integral and $\operatorname{gcd}\left(s_{n}, b s_{n-1}\right)=1$, both of fractions cannot be the same unless $\epsilon_{0}$ becomes integral.

We assume that $b= \pm 1$ in stating two theorems. This assumption guarantees that $\epsilon_{1}$ becomes integral after deciding $\epsilon_{n}, \epsilon_{n-1}, \ldots, \epsilon_{2}$. Otherwise, $\epsilon_{1}$ may not become integral by the method in this paper.

BULlETIN DE LA SOCIÉTÉ MATHÉMATIQUE DE FRANCE 


\section{Proof of Theorem 1.1}

Let $b=1$. Since the continued fraction expansion of $\alpha$ is $\alpha=[a ; a, a, \ldots]$, the sequence $\left(q_{n}\right)_{n}$ also satisfies the recurrence relation $r_{n}=a r_{n-1}+1 \cdot r_{n-2}$, which appeared in the proof of Lemma 2.1(1), with $q_{1}=a$ and $q_{0}=1$. Hence, $s_{n}=q_{n-1}(n \geq 0)$. Thus, we obtain

$$
\begin{aligned}
q_{k} s_{n-1}+p_{k} s_{n} & =q_{k} q_{n-2}+q_{k+1} q_{n-1} \\
& =q_{k} q_{n-2}+q_{k+1}\left(a q_{n-2}+q_{n-3}\right) \\
& =\left(a q_{k+1}+q_{k}\right) q_{n-2}+q_{k+1} q_{n-3} \\
& =q_{k+1} q_{n-3}+q_{k+2} q_{n-2}=\cdots \\
& =q_{n+k-2} q_{0}+q_{n+k-1} q_{1}=a q_{n+k-1}+q_{n+k-2}=q_{n+k}
\end{aligned}
$$

and

$$
\begin{aligned}
s_{1}+s_{2}+\cdots+s_{n-1}+s_{n} & =q_{0}+q_{1}+\cdots+q_{n-1} \\
& =\frac{1}{\alpha-\beta}\left(\alpha \frac{\alpha^{n}-1}{\alpha-1}-\beta \frac{\beta^{n}-1}{\beta-1}\right) \\
& =\frac{1}{a} \frac{\alpha^{n+1}-\beta^{n+1}+\alpha^{n}-\beta^{n}-(\alpha-\beta)}{\alpha-\beta} \\
& =\frac{1}{a}\left(s_{n+1}+s_{n}-1\right)=\frac{1}{a}\left(q_{n}+q_{n-1}-1\right) .
\end{aligned}
$$

Therefore, the equation (5) can be written as

$$
-(-1)^{n-1} s_{1} \epsilon_{1}-(-1)^{n-2} s_{2} \epsilon_{2}-\cdots+s_{n-1} \epsilon_{n-1}-s_{n} \epsilon_{n}=q_{n+k} .
$$

Since we would like to choose $\epsilon_{n}, \epsilon_{n-1}, \ldots, \epsilon_{1}$ so that $\max _{i}\left|\epsilon_{i}\right|$ is as small as possible, it is sufficient to consider the equation

$$
s_{1}\left|\epsilon_{1}\right|+s_{2}\left|\epsilon_{2}\right|+\cdots+s_{n-1}\left|\epsilon_{n-1}\right|+s_{n}\left|\epsilon_{n}\right|=q_{n+k} .
$$

We shall choose $m^{\prime}$ so that

$$
m \geq m^{\prime}=\inf _{n}\left\lceil\frac{q_{k} s_{n-1}+p_{k} s_{n}}{s_{1}+s_{2}+\cdots+s_{n-1}+s_{n}}\right\rceil=\inf _{n}\left\lceil\frac{a q_{n+k}}{q_{n}+q_{n-1}-1}\right\rceil
$$

and take $\left|\epsilon_{n}\right|=m^{\prime}$. This $m^{\prime}$ is the lower bound of $m$ because if we take $\left|\epsilon_{n}\right| \leq m^{\prime}-1$, then at least one of $\left|\epsilon_{1}\right|, \ldots,\left|\epsilon_{n-1}\right|$ must exceed $m^{\prime}$.

We need the following lemma to complete the proof of Theorem 1.1.

Lemma 3.1. - The sequence

$$
\frac{a q_{n+k}}{q_{n}+q_{n-1}-1}
$$

is a monotone decreasing function in $n$, tending to $\alpha^{k}(\alpha-1)$.

TOME $130-2002-\mathrm{N}^{\mathrm{O}} 1$ 
REMARK 3.2. - In general, if $a+b>1$ and $a+1>b>0$ then

$$
\frac{q_{k} b s_{n-1}+p_{k} s_{n}}{s_{n+1}+s_{n}-b^{n}} \downarrow \frac{b q_{k}+\alpha_{k}}{\alpha(\alpha+1)} \quad(n \rightarrow \infty) \text {. }
$$

Proof of Lemma 3.1. - Since $|\beta|<1<|\alpha|$, we have

$$
\begin{aligned}
\frac{a q_{n+k}}{q_{n}+q_{n-1}-1} & =\frac{a\left(\alpha^{k}-(\beta / \alpha)^{n+1} \beta^{k}\right)}{1-(\beta / \alpha)^{n+1}+1 / \alpha-\beta / \alpha^{n+1}-(\alpha-\beta) / \alpha^{n+1}} \\
& \longrightarrow \frac{a \alpha^{k}}{1+1 / \alpha}=\alpha^{k}(\alpha-1) \quad(n \rightarrow \infty) .
\end{aligned}
$$

Putting

$$
f(n)=\frac{q_{k} b s_{n-1}+p_{k} s_{n}}{s_{n+1}+s_{n}-b^{n}} \quad(n=1,2, \ldots),
$$

show $f(n)>f(n+1)$, which is equivalent to

(8) $\left(q_{n+k} q_{n+1}-q_{n+k+1} q_{n}\right)+\left(q_{n+k} q_{n}-q_{n+k+1} q_{n-1}\right)-q_{n+k}+q_{n+k+1}>0$.

By Lemma 2.1(2) with $q_{n-1}=s_{n}$, for $k=0,1,2, \ldots$

$$
\left(q_{n+k} q_{n+1}-q_{n+k+1} q_{n}\right)+\left(q_{n+k} q_{n}-q_{n+k+1} q_{n-1}\right)=(-1)^{n-1} q_{k-1}+(-1)^{n} q_{k} .
$$

If $n$ is even, then the left-hand side of $(8)=q_{k}-q_{k-1}+q_{n+k+1}-q_{n+k}>0$.

If $n$ is odd, then the left-hand side of $(8) \geq q_{k+2}-q_{k+1}-q_{k}+q_{k-1} \geq q_{k-1}>0$.

When $k=-1$, the left-hand side of $(8)=q_{n}-q_{n-1}+(-1)^{n-1} \geq 0$. The equality sign holds only when $a=b=1$ and $n=2$.

We can find the sequence $\epsilon_{n-1}, \ldots, \epsilon_{1}$ and $\epsilon_{0}$ one after another, as follows. Concerning the equation (5) or (6) we choose $n^{\prime}$ as the least integer satisfying

$$
-\epsilon_{n^{\prime}}=\left|\epsilon_{n^{\prime}}\right|=\left\lceil\frac{q_{n^{\prime}+k}}{s_{1}+s_{2}+\cdots+s_{n^{\prime}}}\right\rceil=m^{\prime} \quad\left(=\inf _{n}\left\lceil\frac{a q_{n+k}}{q_{n}+q_{n-1}-1}\right\rceil\right) .
$$

We can take any integer $n$ with $n \geq n^{\prime}$. It is, however, simple and easy to take $n=n^{\prime}$ in the practical applications (see examples in section 5). Concerning the new equation

$$
-(-1)^{n-1} s_{1} \epsilon_{1}-(-1)^{n-2} s_{2} \epsilon_{2}-\cdots-s_{n-2} \epsilon_{n-2}+s_{n-1} \epsilon_{n-1}=q_{n+k}+s_{n} \epsilon_{n}
$$

or

$$
\left|\epsilon_{1}\right|+a\left|\epsilon_{2}\right|+\cdots+s_{n-1}\left|\epsilon_{n-1}\right|=q_{n+k}-s_{n}\left|\epsilon_{n}\right|,
$$

we take

$$
\epsilon_{n-1}=\left\lceil\frac{q_{n+k}-s_{n}\left|\epsilon_{n}\right|}{s_{1}+s_{2}+\cdots+s_{n-1}}\right\rceil .
$$

We repeat the similar step. For $i=n-1, n-2, \ldots, 2$ we take

$$
(-1)^{n-i+1} \epsilon_{i}=\left|\epsilon_{i}\right|=\left\lceil\frac{q_{n+k}-s_{n}\left|\epsilon_{n}\right|-\cdots-s_{i+1}\left|\epsilon_{i+1}\right|}{s_{1}+s_{2}+\cdots+s_{i}}\right\rceil .
$$

BULLETIN DE LA SOCIÉTÉ MATHÉMATIQUe DE FRANCE 
Since $\left|(-1)^{n-1} s_{1}\right|=1, \epsilon_{1}$ is also chosen as an integer with $\left|\epsilon_{1}\right| \leq\left|\epsilon_{2}\right| \leq \cdots \leq$ $\left|\epsilon_{n}\right| \leq m$. Notice that if $q_{n+k}$, the right-hand side of (6), is small, $\left|\epsilon_{1}\right|$ may become negative in this algorithm (see [2], p. 21). But since $q_{n+k}>\bar{d}_{n}$, where

$$
\bar{d}_{1}=-1, \quad \bar{d}_{i}=\left\lceil\frac{\bar{d}_{i-1}+s_{i}}{\sum_{j=1}^{i-1} s_{j}}\right\rceil s_{i}+\bar{d}_{i-1} \quad(i=2, \ldots, n),
$$

such a troublesome case does never happen and this algorithm works properly (see [2], Theorem 1). We omit this proof because it is clear if $k$ is large; it is manually checked if $k$ is small.

Finally, by substituting these $\epsilon_{1}, \epsilon_{2}, \ldots, \epsilon_{n}$ into one of the equations (3) and (4) one finds $\epsilon_{0}$, which is an integer because $\operatorname{gcd}\left(s_{n}, s_{n-1}\right)=\operatorname{gcd}(a, 1)=1$. Note that $\left|\epsilon_{0}\right| \leq m^{\prime}$ because from (3) and (5)

$$
\epsilon_{0} s_{n}=q_{k}-\left|\epsilon_{n-1}\right| s_{1}+\left|\epsilon_{n-2}\right| s_{2}-\cdots+(-1)^{n-1}\left|\epsilon_{1}\right| s_{n-1} .
$$

Thus, when $n$ is odd,

$$
\begin{aligned}
\epsilon_{0} & \leq \frac{1}{s_{n}}\left(q_{k}+\frac{a q_{n+k}}{q_{n}+q_{n-1}-1}\left(-s_{1}+s_{2}-\cdots-s_{n-2}+s_{n-1}\right)\right) \\
& =\frac{1}{q_{n-1}}\left(q_{k}+\frac{a q_{n+k}}{q_{n}+q_{n-1}-1} \frac{s_{n}-s_{n-1}-1}{a}\right) \\
& =\frac{q_{n+k}-q_{n+k-1}+q_{k}-q_{k-1}}{q_{n}+q_{n-1}-1} \leq \frac{a q_{n+k}}{q_{n}+q_{n-1}-1} .
\end{aligned}
$$

When $n$ is even,

$$
\begin{aligned}
\epsilon_{0} & \geq \frac{1}{s_{n}}\left(q_{k}+\frac{a q_{n+k}}{q_{n}+q_{n-1}-1}\left(-s_{1}+s_{2}-\cdots+s_{n-2}-s_{n-1}\right)\right) \\
& =\frac{1}{q_{n-1}}\left(q_{k}+\frac{a q_{n+k}}{q_{n}+q_{n-1}-1} \frac{-s_{n}+s_{n-1}-1}{a}\right) \\
& =\frac{-q_{n+k}+q_{n+k-1}+q_{k}-q_{k-1}}{q_{n}+q_{n-1}-1} \geq-\frac{a q_{n+k}}{q_{n}+q_{n-1}-1} .
\end{aligned}
$$

Theorem 1.1 includes the case for the golden number $\alpha=[1 ; 1,1, \ldots]=$ $\frac{1}{2}(1+\sqrt{5})\left(\right.$ see $[8]$, Theorem 3). Put $a=1$. Since $q_{k-1}=F_{k}$ and $\alpha^{k}(\alpha-1)=$ $\alpha^{k-1}$, where $\left(F_{k}\right)$ is the Fibonacci sequence defined by

$$
F_{0}=0, \quad F_{1}=1, \quad F_{k}=F_{k-1}+F_{k-2} \quad(k=2,3, \ldots),
$$

we have

$$
\ell^{m}(\alpha)=\left|q_{k-1} \alpha-q_{k}\right|=\left|F_{k} \alpha-F_{k+1}\right|
$$

if $\alpha^{k-2}<m \leq \alpha^{k-1}$.

Notice that $\alpha^{k}(\alpha-1)=1$ and

$$
\left\lceil\frac{a q_{n+k}}{q_{n}+q_{n-1}-1}\right\rceil=2 \quad(n=1,2, \ldots)
$$

TOME $130-2002-\mathrm{N}^{\mathrm{O}} 1$ 
when $\alpha=\frac{1}{2}(1+\sqrt{5})$ and $k=1$. Therefore, the equality sign on the right-hand side is necessary in Theorem 1.1.

\section{Proof of Theorem 1.2}

Let $b<0$. Put $b^{\prime}=-b>0$ for convenience. By $\alpha \beta=b^{\prime}$ and $\alpha+\beta=a$ we have

$$
\begin{aligned}
b^{\prime n-1} s_{1}+b^{\prime n-2} s_{2}+\cdots+ & b^{\prime} s_{n-1}+s_{n}=\frac{1}{\alpha-\beta}\left(\alpha \frac{\alpha^{n}-b^{\prime n}}{\alpha-b^{\prime}}-\beta \frac{\beta^{n}-b^{\prime n}}{\beta-b^{\prime}}\right) \\
= & \frac{1}{a-b^{\prime}-1} \frac{\alpha^{n+1}-\beta^{n+1}-\alpha^{n}+\beta^{n}-b^{\prime n}(\alpha-\beta)}{\alpha-\beta} \\
= & \frac{1}{a+b-1}\left(s_{n+1}-s_{n}-(-b)^{n}\right) .
\end{aligned}
$$

Hence, the equation (5) becomes

$$
-b^{\prime n-1} s_{1} \epsilon_{1}-b^{\prime n-2} s_{2} \epsilon_{2}-\cdots-b^{\prime} s_{n-1} \epsilon_{n-1}-s_{n} \epsilon_{n}=-q_{k} b^{\prime} s_{n-1}+p_{k} s_{n}
$$

or

$$
b^{\prime n-1}\left|\epsilon_{1}\right|+b^{\prime n-2} a\left|\epsilon_{2}\right|+\cdots+b^{\prime} s_{n-1}\left|\epsilon_{n-1}\right|+s_{n}\left|\epsilon_{n}\right|=-q_{k} b^{\prime} s_{n-1}+p_{k} s_{n} .
$$

We shall choose $m^{\prime}$ so that

$$
\begin{aligned}
m \geq m^{\prime}=\left|\epsilon_{n}\right| & =\inf _{n}\left\lceil\frac{q_{k} b s_{n-1}+p_{k} s_{n}}{b^{\prime n-1} s_{1}+b^{\prime n-2} s_{2}+\cdots+b^{\prime} s_{n-1}+s_{n}}\right\rceil \\
& =\inf _{n}\left\lceil(a+b-1) \frac{q_{k} b s_{n-1}+p_{k} s_{n}}{s_{n+1}-s_{n}-(-b)^{n}}\right\rceil .
\end{aligned}
$$

We need Lemma 4.1 and Lemma 4.3 to complete the proof of Theorem 1.2.

LEMMA 4.1. - If $a \geq 3$ and $b=-1$, then

$$
\frac{-q_{k} s_{n-1}+p_{k} s_{n}}{s_{n+1}-s_{n}-1}
$$

is a monotone decreasing function in $n$, tending to $\left(-q_{k}+\alpha p_{k}\right) /(\alpha(\alpha-1))$.

REMARK 4.2. - In general, if $a+b>1$ and $b<0$ then

$$
\frac{q_{k} b s_{n-1}+p_{k} s_{n}}{s_{n+1}-s_{n}-(-b)^{n}} \downarrow \frac{b q_{k}+\alpha p_{k}}{\alpha(\alpha-1)} \quad(n \rightarrow \infty) \text {. }
$$

Lemma 4.3. - When $b=-1$, for $i=0,1,2, \ldots$

$$
\begin{array}{ll}
p_{2 i-1}=s_{i+1}, & p_{2 i-2}=s_{i+1}-s_{i}, \\
q_{2 i-1}=s_{i}, & q_{2 i-2}=s_{i}-s_{i-1} .
\end{array}
$$

BULLETIN DE LA SOCIÉTÉ MATHÉMATIQUE DE FRANCE 
Moreover,

$$
-q_{k} s_{n-1}+p_{k} s_{n}= \begin{cases}q_{2 n+k}, & \text { if } k \text { is odd } \\ q_{2 n+k+1}-q_{2 n+k-1}, & \text { if } k \text { is even }\end{cases}
$$

Proof of Lemma 4.1. - One has

$$
\begin{aligned}
& \frac{-q_{k} s_{n-1}+p_{k} s_{n}}{s_{n+1}-s_{n}-1}=\frac{-q_{k}\left(\alpha^{n-1}-\beta^{n-1}\right)+p_{k}\left(\alpha^{n}-\beta^{n}\right)}{\alpha^{n+1}-\beta^{n+1}-\alpha^{n}+\beta^{n}-(\alpha-\beta)} \\
& \longrightarrow \frac{-q_{k} / \alpha+p_{k}}{\alpha-1}=\frac{-q_{k}+\alpha p_{k}}{\alpha(\alpha-1)} \quad(n \rightarrow \infty) .
\end{aligned}
$$

Putting

$$
g(n)=\frac{-q_{k} s_{n-1}+p_{k} s_{n}}{s_{n+1}-s_{n}-1} \quad(n=1,2, \ldots),
$$

show $g(n)>g(n+1)$, which is equivalent to

$$
\begin{array}{r}
\left(-q_{k} s_{n-1}+p_{k} s_{n}\right)\left(s_{n+2}-s_{n+1}-1\right)-\left(-q_{k} s_{n}+p_{k} s_{n+1}\right)\left(s_{n+1}-s_{n}-1\right) \\
=p_{k}\left(s_{n+1}-s_{n}-1\right)-q_{k}\left(\left(s_{n}-s_{n-1}\right)-(a-1)\right)>0
\end{array}
$$

by Lemma 2.1(2).

Since the continued fraction expansion of $\alpha$ is $\alpha=[a-1 ; 1, \ldots]$, we get

$$
a-1=\frac{p_{0}}{q_{0}} \leq \frac{p_{k}}{q_{k}} \leq \frac{p_{1}}{q_{1}}=a \quad(k=0,1,2, \ldots) .
$$

Thus, we have

$$
\begin{aligned}
p_{k}\left(s_{n+1}\right. & \left.-s_{n}-1\right)-q_{k}\left(\left(s_{n}-s_{n-1}\right)-(a-1)\right) \\
& \geq q_{k}\left((a-1)\left(s_{n+1}-s_{n}-1\right)-\left(s_{n}-s_{n-1}\right)+(a-1)\right) \\
& >q_{k}\left(\left(s_{n+1}-s_{n}\right)-\left(s_{n}-s_{n-1}\right)\right) \\
& =q_{k}\left(\alpha^{n+1}-\alpha^{n}\right)>0 .
\end{aligned}
$$

When $k=-1$, it is clear that $g(n)>g(n+1)$ by $p_{-1}=1$ and $q_{-1}=0$.

Proof of Lemma 4.3. - When $b=-1, \alpha=[a-1 ; 1, a-2,1, a-2,1, a-2, \ldots]$. Thus, $s_{0}=0, s_{1}=1, s_{2}=a, s_{3}=a^{2}-1, s_{4}=a^{3}-2 a, \ldots$ and

$$
\frac{p_{0}}{q_{0}}=\frac{a-1}{1}, \quad \frac{p_{1}}{q_{1}}=\frac{a}{1}, \quad \frac{p_{2}}{q_{2}}=\frac{a^{2}-a-1}{a-1}, \quad \frac{p_{3}}{q_{3}}=\frac{a^{2}-1}{a}, \ldots .
$$

Then, the first part is followed by the induction.

TOME $130-2002-\mathrm{N}^{\mathrm{O}} 1$ 
By using the first part we obtain

$$
\begin{aligned}
-q_{2 i-1} s_{n-1}+p_{2 i-1} s_{n} & =-s_{i} s_{n-1}+s_{i+1} s_{n} \\
& =\left(a s_{i}-s_{i-1}\right) s_{n}-s_{i} s_{n-1}=s_{i}\left(a s_{n}-s_{n-1}\right)-s_{i-1} s_{n} \\
& =s_{i} s_{n+1}-s_{i-1} s_{n}=s_{i-1} s_{n+2}-s_{i-2} s_{n+1}=\cdots \\
& =s_{1} s_{n+i}-s_{0} s_{n+i-1}=s_{n+1}=q_{2 n+k}
\end{aligned}
$$

if $k=2 i-1$, and

$$
\begin{aligned}
-q_{2 i} s_{n-1}+p_{2 i} s_{n} & =\left(-s_{i+1} s_{n-1}+s_{i+2} s_{n}\right)-\left(s_{i+1} s_{n}-s_{i} s_{n-1}\right) \\
& =s_{n+i+1}-s_{n+i}=q_{2 n+k+1}-q_{2 n+k-1}
\end{aligned}
$$

if $k=2 i$.

Put $b=-1$. If $k=2 i-1$, then

$$
m^{\prime}=\inf _{n}\left\lceil(a-2) \frac{q_{2 n+k}}{q_{2 n+1}-q_{2 n-1}-1}\right\rceil
$$

and

$$
\begin{aligned}
\frac{a+b-1}{\alpha(\alpha-1)}\left(b q_{k}+\alpha p_{k}\right) & =\frac{a-2}{\alpha(\alpha-1)}\left(-s_{i}+\alpha s_{i+1}\right) \\
& =\frac{(a-2) \alpha^{i+1}}{\alpha(\alpha-1)}=(\alpha-a+1) \alpha^{i} .
\end{aligned}
$$

If $k=2 i$, then

$$
m^{\prime}=\inf _{n}\left\lceil(a-2) \frac{q_{2 n+k+1}-q_{2 n+k-1}}{q_{2 n+1}-q_{2 n-1}-1}\right\rceil
$$

and

$$
\begin{aligned}
\frac{a+b-1}{\alpha(\alpha-1)}\left(b q_{k}+\alpha p_{k}\right) & =\frac{a-2}{\alpha(\alpha-1)}\left(\left(\alpha s_{i+2}-s_{i+1}\right)-\left(\alpha s_{i+1}-s_{i}\right)\right) \\
& =(a-2) \alpha^{i}
\end{aligned}
$$

The process to determine $\left|\epsilon_{n-1}\right|,\left|\epsilon_{n-2}\right|, \ldots,\left|\epsilon_{2}\right|,\left|\epsilon_{1}\right|$ and $\epsilon_{0}$ is similar to the case where $b=1$.

Notice that $m^{\prime}=\lceil a-2\rceil=a-2$ and

$$
\left\lceil(a-2) \frac{q_{2 n+1}-q_{2 n-1}}{q_{2 n+1}-q_{2 n-1}-1}\right\rceil=a-1 \quad(n=1,2, \ldots)
$$

when $k=0$. Therefore, the equality sign is necessary in Theorem 1.2.

BULLETIN DE LA SOCIÉTÉ MATHÉMATIQUE DE FRANCE 


\section{Examples}

ExAmple 5.1. - Take $\alpha=\sqrt{2}+1=[2 ; 2,2, \ldots]$. Then by Theorem 1.1 we obtain that

$$
\ell^{m}(\alpha)= \begin{cases}1 & \text { if } m=1 ; \\ \left|q_{0} \alpha-p_{0}\right|=\alpha-2 & \text { if } m=2,3 \\ \left|q_{1} \alpha-p_{1}\right|=5-2 \alpha & \text { if } 4 \leq m \leq 8 \\ \left|q_{2} \alpha-p_{2}\right|=5 \alpha-12 & \text { if } 9 \leq m \leq 19 ; \\ \left|q_{3} \alpha-p_{3}\right|=29-12 \alpha & \text { if } 20 \leq m \leq 48 \\ \left|q_{4} \alpha-p_{4}\right|=29 \alpha-70 & \text { if } 49 \leq m \leq 115\end{cases}
$$

We shall check $\ell^{49}(\alpha)=29 \alpha-70$. Find the least integer $n$ satisfying

$$
\left\lceil\frac{q_{n+4}}{q_{0}+\cdots+q_{n-1}}\right\rceil=49 .
$$

Since

$$
\left\lceil\frac{q_{8}}{q_{0}+\cdots+q_{3}}\right\rceil=50 \text { and }\left\lceil\frac{q_{9}}{q_{0}+\cdots+q_{4}}\right\rceil=49,
$$

we can take $n=5$ (of course, it is possible to take $n=6,7, \ldots$ too). Thus, substituting $q_{1}=2, q_{2}=5, q_{3}=12, q_{4}=29$ and $q_{9}=2378$ into $-\epsilon_{1}+q_{1} \epsilon_{2}-$ $q_{2} \epsilon_{3}+q_{3} \epsilon_{4}-q_{4} \epsilon_{5}=q_{9}$, we get the equation

$$
-\epsilon_{1}+2 \epsilon_{2}-5 \epsilon_{3}+12 \epsilon_{4}-29 \epsilon_{5}=2378 .
$$

Thus, $-\epsilon_{5}=\lceil 2378 /(1+2+5+12+29)\rceil=\lceil 48.53 \ldots\rceil=49$.

By $-\epsilon_{1}+2 \epsilon_{2}-5 \epsilon_{3}+12 \epsilon_{4}=957$ we take

$$
\epsilon_{4}=\left\lceil\frac{957}{1+2+5+12}\right\rceil=\lceil 47.85\rceil=48 .
$$

By $-\epsilon_{1}+2 \epsilon_{2}-5 \epsilon_{3}=381$ we take

$$
-\epsilon_{3}=\left\lceil\frac{381}{1+2+5}\right\rceil=\lceil 47.625\rceil=48 .
$$

By $-\epsilon_{1}+2 \epsilon_{2}=141$ we take

$$
\epsilon_{2}=\left\lceil\frac{141}{1+2}\right\rceil=47
$$

Hence, $-\epsilon_{1}=47$. Now, substitute the known values into $\epsilon_{0} q_{3}+\epsilon_{1} q_{2}+\epsilon_{2} q_{1}+$ $\epsilon_{3} q_{0}+\epsilon_{5}=-q_{5}\left(\right.$ or $\left.\epsilon_{0} q_{4}+\epsilon_{1} q_{3}+\epsilon_{2} q_{2}+\epsilon_{3} q_{1}+\epsilon_{4}=q_{4}\right)$ we get

$$
12 \epsilon_{0}-47 \cdot 5+47 \cdot 2-48 \cdot 1-49=-70,
$$

TOME $130-2002-\mathrm{N}^{\mathrm{O}} 1$ 
yielding $\epsilon_{0}=14$. In fact,

$$
\begin{aligned}
y & =14 \alpha^{5}-47 \alpha^{4}+47 \alpha^{3}-48 \alpha^{2}+48 \alpha-49 \\
& =14(29 \alpha+12)-47(12 \alpha+5)+47(5 \alpha+2)-48(2 \alpha+1)+48 \alpha-49 \\
& =29 \alpha-70 .
\end{aligned}
$$

EXAMPLE 5.2. - Let $a=3$ and $b=-1$. Then $\alpha=\frac{1}{2}(3+\sqrt{5})=[2 ; 1,1, \ldots]$, satisfying $\alpha^{2}=3 \alpha-1$. Notice that

$$
\frac{p_{0}}{q_{0}}=\frac{2}{1}, \quad \frac{p_{1}}{q_{1}}=\frac{3}{1}, \quad \frac{p_{2}}{q_{2}}=\frac{5}{2}, \quad \frac{p_{3}}{q_{3}}=\frac{8}{3}, \quad \frac{p_{4}}{q_{4}}=\frac{13}{5}, \quad \frac{p_{5}}{q_{5}}=\frac{21}{8}, \ldots
$$

and

$$
s_{0}=0, s_{1}=1, s_{2}=3, s_{3}=8, s_{4}=21, s_{5}=55, s_{6}=144, s_{7}=377, \ldots
$$

Then by Theorem 1.2 the least integer $m$ giving $\left|q_{4} \alpha-p_{4}\right|=5 \alpha-13$ is

$$
m \geq m^{\prime}=\left\lceil(3-2) \alpha^{2}\right\rceil=\left\lceil\frac{3 \sqrt{5}+7}{2}\right\rceil=\lceil 6.854 \ldots\rceil=7 .
$$

Find the least integer $n$ satisfying

$$
\left\lceil(a-2) \frac{-q_{k} s_{n-1}+p_{k} s_{n}}{s_{n+1}-s_{n}-1}\right\rceil=49
$$

though we can take an arbitrary large $n$. Since

$$
\left\lceil(3-2) \frac{-5 \cdot 8+13 \cdot 21}{55-21-1}\right\rceil=\lceil 7.06 \ldots\rceil=8 \text { for } \quad n=4
$$

and

$$
\left\lceil(3-2) \frac{-5 \cdot 21+13 \cdot 55}{144-55-1}\right\rceil=\lceil 6.93 \ldots\rceil=7 \text { for } n=5,
$$

we can take $n=5$. Thus, substituting the known quantities into $-\epsilon_{1}-q_{1} \epsilon_{2}-$ $q_{2} \epsilon_{3}-q_{3} \epsilon_{4}-q_{4} \epsilon_{5}=-q_{4} s_{4}+p_{4} s_{5}$, we get the equation

$$
-\epsilon_{1}-3 \epsilon_{2}-8 \epsilon_{3}-21 \epsilon_{4}-55 \epsilon_{5}=610 \text {. }
$$

Thus, $-\epsilon_{5}=\lceil 610 /(1+3+8+21+55)\rceil=\lceil 6.9 \ldots\rceil=7$.

By $-\epsilon_{1}-3 \epsilon_{2}-8 \epsilon_{3}-21 \epsilon_{4}=225$ we take

$$
-\epsilon_{4}=\left\lceil\frac{225}{1+3+8+21}\right\rceil=\lceil 6.8 \ldots\rceil=7 .
$$

By $-\epsilon_{1}-3 \epsilon_{2}-8 \epsilon_{3}=78$ we take

$$
-\epsilon_{3}=\left\lceil\frac{78}{1+3+8}\right\rceil=\lceil 6.5\rceil=7 .
$$

By $-\epsilon_{1}-3 \epsilon_{2}=22$ we take

$$
-\epsilon_{2}=\left\lceil\frac{22}{1+3}\right\rceil=\lceil 5.5\rceil=6 .
$$

BULLETIN DE LA SOCIÉTÉ MATHÉMATIQUE DE FRANCE 
Hence, $-\epsilon_{1}=4$. Now, substitute the known values into $-\left(\epsilon_{0} s_{4}+\epsilon_{1} s_{3}+\epsilon_{2} s_{2}+\right.$ $\left.\epsilon_{3} s_{1}\right)+\epsilon_{5}=-p_{4}\left(\right.$ or $\left.\epsilon_{0} s_{5}+\epsilon_{1} s_{4}+\epsilon_{2} s_{3}+\epsilon_{3} s_{2}+\epsilon_{4}=q_{4}\right)$ we get

$$
-21 \epsilon_{0}+4 \cdot 8+6 \cdot 3+7 \cdot 1-7=-13,
$$

yielding $\epsilon_{0}=3$. In fact,

$$
\begin{aligned}
y & =3 \alpha^{5}-4 \alpha^{4}-6 \alpha^{3}-7 \alpha^{2}-7 \alpha-7 \\
& =3(55 \alpha-21)-4(21 \alpha-8)-6(8 \alpha-3)-7(3 \alpha-1)-7 \alpha-7 \\
& =5 \alpha-13 \quad(=0.09016 \ldots) .
\end{aligned}
$$

\section{BIBLIOGRAPHY}

[1] Y. BugEAUd - On a property of Pisot numbers and related questions, Acta Math. Hungar. 73 (1996), p. 33-39.

[2] M. Djawadi \& G. Hofmeister - Linear diophantine problems, Arch. Math. (Basel) 66 (1996), p. 19-29.

[3] P. Erdős, I. Joó \& M. Joó - On a problem of Tamás Varga, Bull. Soc. Math. France 120 (1992), p. 507-521.

[4] P. ERdős, I. Joó \& V. Komornik - Characterization of the unique expressions $1=\sum q^{-n_{i}}$ and related problems, Bull. Soc. Math. France 118 (1990), p. 377-390.

[5] On the sequence of numbers of the form $\epsilon_{0}+\epsilon_{1} q+\cdots+\epsilon_{n} q^{n}$, $\epsilon_{i} \in\{0,1\}$, Acta Arith. 83 (1998), p. 201-210.

[6] P. ERdős \& V. Komornik - On developments in noninteger bases, Acta Math. Hungar. 79 (1998), p. 57-83.

[7] V. KOMORNiK \& P. LORETI - Unique developments in non-integer bases, Amer. Math. Monthly 105 (1998), p. 636-639.

[8] V. Komornik, P. Loreti \& M. Pedicini - An approximation property of Pisot numbers, J. Number Theory 80 (2000), p. 218-237.

[9] T. VAn RAVEnstein - The three gap theorem (Steinhaus conjecture), J. Austral. Math. Soc. Ser. A 45 (1988), p. 360-370.

[10] W. Schmidt - Diophantine approximation, Lecture Notes in Math., vol. 785, Springer-Verlag, 1980. 